\title{
Epidemiological and virological analysis of couples infected with hepatitis $\mathrm{C}$ virus
}

\author{
H Zylberberg, V Thiers, D Lagorce, G Squadrito, F Leone, P Berthelot, C Bréchot, S Pol
}

\begin{abstract}
Background-If transmission of hepatitis $C$ virus $(\mathrm{HCV})$ infection through parenteral exposure is well documented, sexual transmission of $\mathrm{HCV}$ is still debated.

Aims-To perform extensive epidemiological and virological analysis in 24 couples in which each spouse was antiHCV positive in order to delineate more precisely potential sexual transmission of HCV.
\end{abstract}

Patients-Twenty four couples in which each partner was anti-HCV positive. These 48 spouses were recruited in a liver unit by regular screening of spouses of index patients.

Methods-All 48 spouses completed an epidemiological questionnaire on risk factors for HCV. Qualitative detection of serum HCV RNA and determination of HCV type by genotyping and serotyping were performed. Sequence analysis of HCV strains by phylogenetic analysis was carried out in seven couples with concordant genotypes.

Results-The mean (SD) partnership duration was 12 (10) years. Serum HCV RNA was detected in both partners in 18 of the couples $(75 \%)$ and in only one partner in six of the couples $(25 \%)$. HCV typing showed concordant genotypes in 12 couples $(50 \%)$, discordant genotypes in seven $(29 \%)$, and in the other five couples (21\%) only one spouse could be genotyped. Of the 48 spouses, 33 had a major risk factor for $\mathrm{HCV}$ transmission such as transfusion $(n=6)$ and intravenous drug use $(n=27)$. Eleven of the 12 couples infected with the same HCV genotype had at least one parenteral risk factor for viral transmission in both spouses. Whatever the genotype concordance, in most couples $(75 \%)$, both spouses showed parenteral risk factors for viral transmission. Sequence analysis of $\mathrm{HCV}$ strains was possible in seven of 12 couples with identical genotype and showed different and identical isolates in four and three couples respectively.

Conclusion-The study emphasises the risk of overestimating the importance of a very low sexual HCV transmission risk as against other, mainly parenteral, risk factors.

(Gut 1999;45:112-116)

Keywords: hepatitis C virus; sexual transmission; liver
The issue of sexual transmission of hepatitis C virus (HCV) is still debated. Conflicting results have indeed been reported, as the rate for such transmission ranges from 0 to $27 \%,{ }^{1-6}$ and it is still unclear whether or not $\mathrm{HCV}$ RNA can be detected in semen. ${ }^{78}$ These discrepancies can be partly explained by a lack of exhaustive epidemiological analysis, parenteral risk factors representing a potentially major epidemiological bias. Finally, although some studies have included sequence analysis, the number of patients tested was still low. ${ }^{2}$ The aim of this work was therefore to delineate more precisely sexual transmission of HCV by performing an extensive epidemiological and virological analysis in 24 couples in which both husband and wife were anti-HCV positive.

\section{Patients and methods}

PATIENTS

We studied 24 couples in which each partner was anti-HCV positive. These couples were randomly recruited in our liver unit by regular screening of spouses of index patients followed in our active file of 1640 anti-HCV positive, including $11.1 \%$ anti-HIV positive patients. Extraconjugual sexual partners were not included in this screening. As many spouses refused anti-HCV determination for various reasons, the exact prevalence of $\mathrm{HCV}$ in spouses of our index subjects was not available. Eight spouses (17\%) in seven couples were anti-HIV positive. Thirty eight of $48(79 \%)$ spouses underwent liver biopsy which showed chronic hepatitis and cirrhosis in $32(84 \%)$ and six $(16 \%)$ respectively. Four additional couples in which both spouses were anti-HCV positive were not included in this study because serum samples were not available for virological analysis in one spouse of the couple.

All 48 subjects responded to a detailed epidemiological questionnaire on risk factors for HCV including history of intravenous drug use, transfusion, acupuncture, tatooing, and suspicion of nosocomial (endoscopy, surgery, mesotherapy) and sporadic transmission (ritual scarring, stay in an endemic geographical area).

VIROLOGICAL METHODS

Serum antibodies to HCV were detected by the second generation HCV enzyme linked immunosorbent assay (ELISA2) and recom-

Abbreviations used in this paper: $\mathrm{HCV}$, hepatitis $\mathrm{C}$ virus; PCR, polymerase chain reaction. d'Hépatologie, Hôpital

F-75015 Paris, France.

Accepted for publication 2 February 1999 
binant immunoblot assay (RIBA2) from Ortho Diagnostic (Raritan, New Jersey, USA), according to the manufacturer's instructions.

Qualitative detection of serum HCV RNA

Briefly, RNA was extracted from $150 \mu \mathrm{l}$ serum, reverse transcribed to cDNA and amplified by polymerase chain reaction (PCR) by a modified nested PCR method with primers located in the 5' non-coding region as previously described. ${ }^{9}$ Amplified products were stained with ethidium bromide and hybridised with a radiolabelled oligonucleotide probe within the amplified sequence.

\section{Genotyping}

Briefly, cDNAs were subjected to amplification. This analysis was based on a restriction fragment length polymorphism assay. Products were digested by three specific restriction endonucleases, Bst $\mathrm{NI}, B s t \mathrm{UI}$, and Sau3a, under the manufacturer's conditions (Biolabs, Beverly, Massachusetts, USA). The fragments were electrophoresed and detected by ethidium bromide staining in comparison with a standard sized DNA marker (100 bp ladder; Promega, Madison, Wisconsin, USA). Their sizes determined the different genotypes 1, 2, 3,4 , and 5 and the subtypes $1 \mathrm{a}$ and $1 \mathrm{~b} .{ }^{9}$

Genotyping by serological assay

A serological assay was performed on the serum of HCV RNA negative spouses. The Murex HCV serotyping 1-6 assay ${ }^{9}$ was used to determine the HCV serotypes. Briefly this analysis is a rapid enzyme immunoassay using synthetic peptides derived from the variable antigenic regions of NS4 of HCV types 1, 2, 3, 4,5 , and 6 .

Sequence and phylogenetic analysis

To compare the HCV strains from the 12 couples with concordant genotype, the NS5b region was chosen for analysis. PCR fragments were characterised by direct sequencing of this region and by comparing the sequences with published sequences using a phylogenetic software package (Phylip-Felsenstein, 1991; University of Washington, Seattle, Washington, USA). Distances between pairs of sequences were estimated using the DNADIST program and further analysed in NEIGHBOR using the neighbor-joining setting. The program Drawgram was used to create a graphic output.

STATISTICAL ANALYSIS

The $\chi^{2}$ and Student's $t$ tests were used for statistical comparisons between couples according to genotype concordance.

\section{Results}

Mean (SD) age of spouses was 40 (13) years (range 25 to 77). The mean (SD) duration of the partnership was 12 (10) years (range 1 to 36). Among the 24 couples, serum HCV RNA was detected in both partners in 18 cases $(75 \%)$ and in only one partner in six (25\%). In couples in which HCV RNA was detected in both spouses, 11 of $18(61 \%)$ had the same genotype while seven of $18(39 \%)$ had different
Table 1 Epidemiological and virological analysis according to genotype concordance in couples

\begin{tabular}{llll}
\hline & $\begin{array}{l}\text { Genotype } \\
\text { discordance } \\
(n=7)\end{array}$ & $\begin{array}{l}\text { Genotype } \\
\text { concordance } \\
(n=12)\end{array}$ & \\
\hline Genotype (n) & $\begin{array}{lll}1 \mathrm{a} / 3 \mathrm{a}(2) \\
1 \mathrm{~b} / 1 \mathrm{a}(1)\end{array}$ & $\begin{array}{l}1 \mathrm{~b}(4)^{\star} \\
3 \mathrm{a}(5)\end{array}$ & \\
& $\begin{array}{l}3 \mathrm{a} / 2 \mathrm{a}(1) \\
1 \mathrm{~b} / 3 \mathrm{a}(1)\end{array}$ & $4(1)$ & \\
& $\begin{array}{l}3 \mathrm{a} / 4(1) \\
1 / 3 \mathrm{a}(1)\end{array}$ & & \\
& $6(85)$ & $11(91)$ & $\mathrm{NS}$ \\
$\begin{array}{c}\text { Parenteral risk factor in } \\
\text { both spouses; } \mathrm{n}(\%)\end{array}$ & & & \\
$\begin{array}{c}\text { Mean (SD) duration of } \\
\text { relationship (years) }\end{array}$ & $7.6(5.8)$ & $13.2(11.3)$ & $\mathrm{NS}$ \\
\hline
\end{tabular}

$\bar{\star}$ Including one couple in which one spouse was only determined by serotyping as type 1 .

genotypes. Genotyping by serological assay was used in the four HCV RNA negative spouses and disclosed one infection by type 1 , and was not conclusive in three cases; in the other two cases, serum was not available for serotyping analysis and thus HCV genotype could not be determined. One couple in which one spouse was infected by genotype $1 \mathrm{~b}$ and the other by a serotype 1 were assumed to be infected by a "concordant" genotype. Thus, among the 24 couples, concordant genotypes were observed in $12(50 \%)$, discordant genotypes in seven $(29 \%)$, and in the remaining five $(21 \%)$ only one spouse could be genotyped, precluding any conclusion about concordance.

Of seven couples in whom at least one partner was anti-HIV positive, three were infected by concordant and two by discordant genotypes; genotype determination was possible in only one spouse for two couples.

The various potential risk factors were analysed in all 48 spouses. Thirty three $(69 \%)$ had a major risk factor of HCV transmission such as transfusion $(n=6)$ and intravenous drug use $(n=27)$. In addition, 13 spouses had other potential risk factors such as history of tatooing, acupuncture, mesotherapy, and history of endoscopy with biopsy or major surgery. Seven spouses (15\%) had at least two parenteral risk factors while nine $(19 \%)$ had no risk factors other than sexual. Of the 19 couples in whom genotype concordance analysis was feasible, 11 of the 12 couples infected with the same HCV genotype had at least one parenteral risk factor for viral transmission in both spouses (including 18 intravenous drug users and two who had received blood transfusions); six of the seven couples infected with different HCV types had at least one parenteral risk factor for viral transmission in both spouses (including six intravenous drug users and two patients who had received blood transfusions (table 1)). In all, 18 of 24 couples $(75 \%)$ had at least one parenteral risk factor for viral transmission in both spouses. In the other six couples, nine spouses had no evidence of parenteral risk factors. Moreover, there was no difference in the distribution of epidemiological risk factors nor in the duration of the relationship according to genotype concordance (table 1).

Owing to the HCV genetic variability, identification of the same HCV genotype in some couples obviously does not imply infection by 


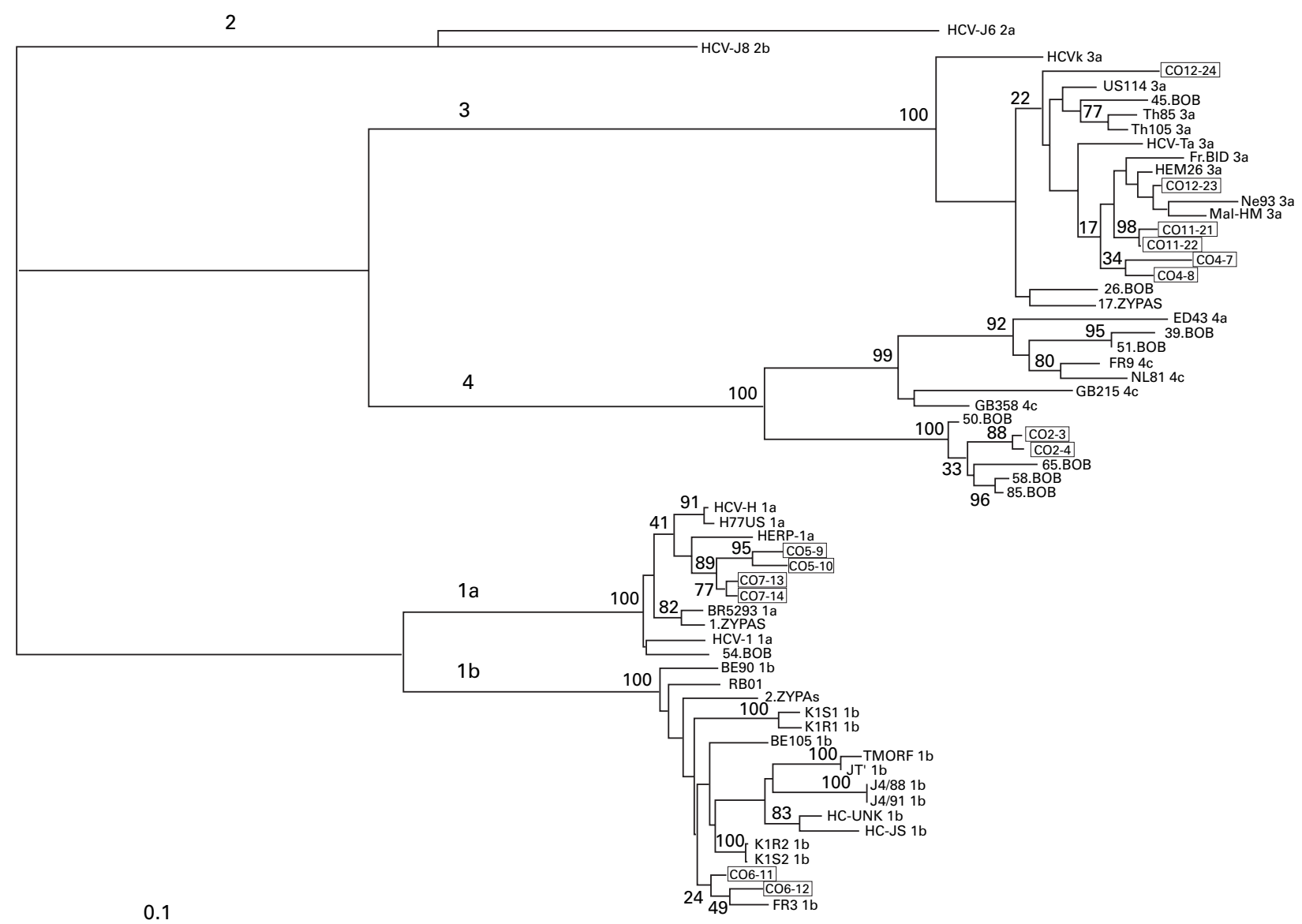

Figure 1 Phylogenetic analysis in seven couples with concordant genotype showing different and identical isolates in four and three couples respectively. The phylogenetic tree is shown of the NS5b region (312 bp fragment; nucleotides 7947-8259) from study cases and previously reported reference sequences. Boxed strains are those recovered in the couples and are identified by the number of the couples from whom they originate- that is, Co12-1 and Co12-2 from couple 12. Accession numbers for the reference isolates used to create this tree have been detailed previously ${ }^{17}$ and identified by the isolate's name.

Furthemore sequences originating from our geographical area were introduced into the phylogenetic analysis (X.BOB). Bootstrap values obtained from 100 replicas are given as percentages at the branching forks.

identical strains. To go further in this issue we analysed the genomic sequence of HCV strains of 12 couples with concordant genotype.

Phylogenetic trees were calculated from the region encompassing nucleotides 7947-8259 of the NS5b region (fig 1). Of the 12 couples infected with concordant genotypes, only seven could be investigated (for some partners the NS5b fragment could not be amplified). The HCV strains from couples 2, 7, and 11 differed by 1 to 3 nucleotides with sequence similarity of $98 \%$ or more (evolutionary distance 0.065 ). These results were further reinforced by $>75 \%$ bootstrap support confirming that these isolates were closely related and suggesting that these spouses were infected by a common source. In contrast, the strains from couples 4, 5,6 , and 12 showed less similarity to each other. They differed by 4 to 15 nucleotides with evolutionary distance 0.0129 . These strains were therefore judged to be unrelated.

To rule out the possibility of PCR contamination, unrelated sera (1.ZYPAS, 2.ZYPAS, 17.ZYPAS belonging to subtypes $1 \mathrm{a}, 1 \mathrm{~b}$, and 3 a respectively) were included and processed together with the samples of interest. These three samples were found on separate branches when compared with the isolates observed in the spouses.
In summary, sequence analysis coupled with phylogenetic analysis showed different isolates in four and identical isolates in three couples (fig 1). Among the couples with identical isolates, risk factors other than sexual were identified in each couple and in three of the four with different isolates.

\section{Discussion}

Our study provides a detailed epidemiological and virological analysis of 24 couples in which both partners were anti-HCV positive and offers evidence, at least in our selected population, that sexual transmission may be mistaken for others factors and thus a careful search for parenteral transmission is needed.

Some $37 \%$ of the couples (in whom concordance genotype analysis was available) did not have the same genotype, which is definitive evidence against sexual transmission. This suggests that the usual major parenteral risk factors (intravenous drug use and blood transfusion) identified in $57 \%$ of these spouses are the cause of the transmission of HCV. In the 12 couples with an identical genotype, sexual transmission of HCV may be hypothesised. However, concordance of genotype within a couple does not provide definitive evidence for sexual transmission. Indeed 
sequence analysis showed that about $60 \%$ of couples analysed with identical genotypes had different isolates. Moreover, identical isolates found in both partners of a couple could be due to parenteral or sporadic transmission and does not systematically prove sexual transmission. Indeed, among the three couples with identical isolates, risk factors other than sexual were identified in each couple, and some subjects reported near exclusive sharing of needles with their partners. Moreover, most if not all of the patients with the same genotype had multiple parenteral risk factors for transmission of $\mathrm{HCV}$, including 19 intravenous drug users and two patients who had received transfusions. It is noteworthy that an absence of well characterised parenteral risk factor for $\mathrm{HCV}$ does not necessarily imply sexual transmission, as inapparent parenteral transmission could be involved in HCV transmission. In addition, there was no difference in the distribution of epidemiological risk factors nor in the length of the relationship according to genotype concordance (table 1 ), and for 16 of the 48 spouses (33\%), the parenteral risk factor of viral transmission preceded the partnership. Along the same lines, it is interesting to note that in 20 stable couples of our cohort in which only one partner was anti-HCV positive, no major parenteral risk factor such as intravenous drug use or history of transfusion was recognised in the anti-HCV negative spouses (data not shown).

A correlation between anti-HCV positivity in spouses of index patients and history of parenteral risk factor has been previously suggested. ${ }^{10}$ Interestingly, the authors failed to demonstrate any link between anti-HCV positivity in spouses of index patients and sexual behaviour (number of sexual partners, anal intercourse, sexually transmitted disease in couples), suggesting that transmission of $\mathrm{HCV}$ did not involve a sexual route. Moreover, in the spouses of HCV infected haemophiliacs, the rate of anti-HCV positivity in wives of index patients has been reported not to exceed the $1 \%$ prevalence observed in the general population. ${ }^{11}$ Finally it is important to note that, despite conflicting results on HCV RNA in semen, no HCV RNA was found in any of the seminal fractions in a large recent study involving 90 subjects. ${ }^{8}$ In contrast, a higher rate of anti-HCV positivity in spouses has been reported in $\operatorname{Japan}^{25}: 27 \%$ and $21 \%$ of HCV infection markers were reported for spouses of 154 and 48 index patients respectively. Concordance in genotypes and homology of nucleotides in HCV sequences in some couples, on the one hand, and correlation between HCV infection in both spouses and duration of marriage $30 \%$ of couples with more than 30 years of marriage were infected as compared with $0 \%$ of those with less than 10 years) on the other hand, were taken as evidence for sexual transmission. ${ }^{2}$ It is noteworthy that a higher prevalence of anti-HCV was observed after more than 30 years of marriage, although sexual activity usually decreases with time. One may therefore hypothesise that a communityacquired or another route of transmission is more likely. This is reinforced by the reported association between HCV infection and traditional practices (acupuncture, "vacuuming") in Asia. ${ }^{12}$ From the same viewpoint, age related factors (either longer period of exposure to potential risk factors or exposure to risk factors that have considerably diminished or disappeared such as the use of non-disposable medical material) may explain the high antiHCV prevalence in 60 year old sexual partners rather than the sexual route. ${ }^{13}$ Finally the level of HCV viraemia may also partly account for different results among the studies. However, it is noteworthy that no major $\mathrm{HCV}$ infection prevalence was found in spouses of patients who had received liver transplantation for $\mathrm{HCV}$ related cirrhosis, in whom the level of viraemia is usually high. ${ }^{14}$

The relatively high frequency of HIV coinfection $(18 \%)$ reflects the high rate of intravenous drug use in our population (six of eight HIV co-infected spouses). However, it is noteworthy that intravenous drug using patients are likely to have intravenous drug using partners, and this underlines the conclusions of our study that sexual transmission may be mistaken for other factors in the absence of extensive epidemiological analysis of risk factors for HCV transmission.

As sexual transmission of $\mathrm{HCV}$ may be favoured in a sexual partner of HCV-HIV co-infected subjects, such a transmission cannot be ruled out in couples in which one of the spouses is HIV infected. ${ }^{15}$ Finally, our findings are in accordance with a recent study in which transmission of $\mathrm{HCV}$ was not observed in couples at risk through sexual contact alone, in contrast with couples in which partners were additionally exposed through intravenous drug use. $^{16}$

We did not test all the sexual partners of our $\mathrm{HCV}$ infected subjects in our series and thus we cannot establish the exact prevalence of HCV positivity among the partners of index cases. The results of our study, however, are in accordance with low sexual transmission of $\mathrm{HCV}$ and underlines the fact that sexual transmission may be mistaken for other factors and therefore a careful search for parenteral transmission is needed. The over-representation of intravenous drug use in our own experience of HCV infected spouses reinforces the hypothesis of parenteral transmission of $\mathrm{HCV}$ even if we cannot exclude the possibility that this over-representation could indeed hide other routes of HCV transmission, and therefore extrapolation of our results to the general population should be made carefully.

1 Bresters D, Mauser-Bunshoten EP, Reesink HW, et al. Sexual transmission of hepatitis $\mathrm{C}$ virus. Lancet 1993;342:210-11.

2 Akahane Y, Kojima M, Sugai Y, et al. Hepatitis C virus infection in spouses of patients with type $\mathrm{C}$ chronic liver disease. Ann Intern Med 1994;120:748-52.

3 Everhart JE, Di Bisceglie AM, Murray LM, et al. Risk for non-A, non-B (type C) hepatitis through sexual or household contact with chronic carriers. Ann Intern Med 1990;112:544-5.

4 Gordon SC, Patel AH, Kulesza GW, et al. Lack of evidence for the heterosexual transmission of hepatitis C. Am $\mathcal{F}$ Gastroenterol 1992;87:1849-51.

5 Kao JH, Chen PJ, Yang PM, et al. Intrafamilial transmission of hepatitis C virus: the important role of infections of hepatitis C virus: the important role
between spouses. F Infect Dis 1992;166:900-3. 
6 Seeff LB, Alter HJ. Spousal transmission of hepatitis C

virus? Ann Intern Med 1994;120:807-9. Hsu $\mathrm{HH}$, Wright $\mathrm{TL}$, Luba $\mathrm{D}$, et al. Failure to detect hepa-
titis $\mathrm{C}$ virus genome in human secretions with the polymerase chain reaction. Hepatology 1991;14:763-7.

8 Persico T, Thiers V, Tuveri R, et al. Detection of hepatitis G/GB-C viral RNA but not HCV RNA in the different semen fractions of infected patients [abstract]. F Hepatol 1996;25:77.

9 Thiers V, Jaffredo F, Tuveri R, et al. Development of a simple restriction fragment length polymorphism (RFLP) based assay for HCV genotyping and comparative analysis with geno.

10 Osmond DH, Padian NS, Sheppard HW, et al. Risk factors for hepatitis $\mathrm{C}$ virus seropositivity in heterosexual couples. ҰAMA 1993;269:361-5.

11 Brackmann SA, Gerritzen A, Oldenburg J, et al. Search for intrafamilial transmission of hepatitis $\mathrm{C}$ virus in hemophiliac patients. Blood 1993;81:1077-82.

12 Kiyosawa K, Tanaka E, Sodeyama T, et al. Transmission of hepatitis $\mathrm{C}$ in an isolated area in Japan: community acquired infection. Gastroenterology 1994;106:1596-602.

13 Diago M, Zapater R, Tuset C, et al. Intrafamily transmission of hepatitis C virus: sexual and non-sexual contacts. $\mathcal{F}$ Hepatol 1996;25:125-8.

14 McCashland TM, Wright TL, Donovan JP, et al. Spousal transmission of hepatitis C after liver transplantation [abstract]. Hepatology 1994;20:134A.

15 Eyster E, Alter JA, Aledort LM, et al. Heterosexual co-transmission of hepatitis $\mathrm{C}$ virus and human immunodeficiency virus. Ann Intern Med 1991;115:764-8.

16 Wyld R, Robertson JR, Brettle RP, et al. Absence of hepatitis $\mathrm{C}$ virus transmission but frequent transmission of HIV-1 from sexual contact with doubly-infected individuals. $\mathcal{F}$ Infect 1998;35:163-6.

17 Stuyver L, Wyseur A, Van Arnhem W, et al. Classification of hepatitis viruses based on phylogenetic analysis of the envelope 1 and non structural $5 \mathrm{~B}$ regions and identification of five additional subtypes. Proc Natl Acad Sci USA 1994;91:10134-8. 